\title{
A HAPTIC BASE HUMAN ROBOT INTERACTION APPROACH FOR ROBOTIC GRIT BLASTING
}

\author{
Pholchai Chotiprayanakul*, Dalong Wang, Ngaiming Kwok and Dikai Liu \\ ARC Centre of Excellence for Autonomous Systems \\ Faculty of Engineering, University of Technology, Sydney \\ Broadway, NSW 2007, Australia \\ pchotipr@eng.uts.edu.au
}

\begin{abstract}
This paper proposes a remote operation method for a robot arm in a complex environment by using the Virtual Force (VF) based approach. A virtual robot arm is manipulated by a steering force, at the end-effecter, which is generated according to the movement of a feedback haptic. A three-dimensional force field $\left(3 \mathrm{D}-\mathrm{F}^{2}\right)$ is employed in collision detection and avoidance. Repulsive forces from the $3 \mathrm{D}-\mathrm{F}^{2}$ are produced and feedback to the haptic device that enables the operator to have a sense of touch on the encountered obstacle and then steer the arm to avoid it. As a result, collision-free poses of the virtual robot arm can then be used to command the real robot. Experiments are conducted in a mock up bridge environment where the real robot arm is steered to target points by the operator. Experiment results have shown successful collision avoidance and emulation of the actual command force and the virtual forces in remote operations.
\end{abstract}

\section{KEYWORDS}

Virtual Force Field, Remote Operation, Human Robot Interaction (HRI), Steel Bridge Maintenance System.

\section{INTRODUCTION}

This paper presents a remote operation method, in the context of Human Robot Interaction (HRI), to manipulate a robot arm in a complex environment by using the Virtual Force (VF) based approach. Remote operations of robotic systems are invaluable to release human operators from hazardous in working environments. For example, in steel bridge maintenance, the grit blasting process often produces stripped dust containing lead, asbestos and other toxic materials that are harmful to workers. Within a complex environment commonly found in bridge structures, it still remains challenging to control a robot arm remotely while avoiding collision with obstacles. Although object-detecting sensors are available, their performances may be hindered in a dusty enclosure for grit blasting. A virtual sense of touch of the obstacles, therefore, would be very valuable to the operator at a remote location. In order to obtain the environmental perception, a force feedback haptic device may be used to render a physical contact between any parts of the robot arm and obstacles. 
The force feedback joystick is a kind of haptic devices, In order to render the contact force, the movement of the haptic's handle is resisted by forces which are generated by a virtual or physical contact. F. Nagata et al. [5] presented a joystick teaching system for industrial robots using fuzzy compliance control. A force sensor, which is attached at the end-effecter of a robot arm, sends the force signals back to the controlling joystick. Their control loop gives the operator the sense of touch when teaching the robot. In typical assembly process, a single force sensor on the end-effecter is used to return a scaled signal to a haptic input device. The scale factor can magnify the sense of touch from a delicate or a micro/nano-scale work [7][6]. Beyond the micro-scale, a physical-contact force sensor can sometime make a huge impact to a tiny work piece. On the other hand, for large-scale work, which is operated by heavy machines such as those found in construction, can also be shrunk to meet the range of human perception.

Virtual reality is frequently used to simulate a virtual robot arm in performing its assigned task. Liao et al. [1] presented a Virtual Force Field, which is defined by a spring model, to shield around an obstacle for collision avoidance purpose. They return a repulsive force on the virtual tool at the end-effecter of the robot arm to the remote control haptic. Motion resistance or payload weight [8], which is measured by a sensor at an actuator such as load cell, force/torque sensor, etc, can be used as a feedback signal to represent the load exerted on the endeffecter. However, these work have addressed cases of only a single force reflection at an operating point. On the other hand, other external forces should be taken into account in the grit blasting operation. Constantinescu et al. [2][3] calculated collision impulses from a passive collision in the virtual world and it was applied to the operator's hand according to the haptic handle's position and the impulse strength. In addition, they presented a force rendering method for a three-link planar manipulator by using repulsive forces and a penalty function [4].

In this work, the interface between a human operator and the robot arm is done in the virtual world, which simulates the real environment by an exploration's method. A steering force, which is derived from an attractive force generation algorithm [9], drives the robot arm's end-effecter on an adjustable controlling plane, in accordance to the coordinate plane of a force feedback joystick. Virtual repulsive forces are created by the three-dimensional force field [10], when the robot arm is moving close to obstacles. The repulsive forces will command, through a sequence of joint angles, the robot arm to avoid any collision and they will be transformed to a force effect of the joystick. After the position controller receives the joint angles and then activates the robot arm, the real robot will travel to the desired pose.

The rest of the paper is arranged as follows. Section 2 describes the grit blasting operation. Section 3 presents the virtual forces, the steering attractive force and the definition of $3 \mathrm{D}$ force field. The dynamic model for the virtual force based approach is given in Section 4. The generation of the sense of touch is described in Section 5. Section 6 presents the experiment results and a conclusion is drawn in Section 7.

\section{GRIT BLASTING OPERATION}

Nowadays, many industrial processes that are dangerous and repetitive have been changed from manual to the autonomous robotic operations. For example, assembling, constructing, fabricating, machining, striping, cleaning and coating. In this paper, the autonomous steel bridge maintenance system, which uses an industrial robot performing grit blasting to remove old paint of a steel bridge (Fig. 1), will be used as an application example.

The robot arm carries a hose and a grit blasting nozzle to blast abrasive media to clean metal structure surfaces. In order to operate a robot arm, a force feedback joystick or other haptic devices will be used that could increase human perception. Different from the teaching pendent, the use of joystick could release the demand for operators with robot control skills. However, the control algorithm designed for a joystick is complicated by un-equal degrees of freedom between the joystick and manipulability of robot arm. Moreover, the operating under complex environment imposes added difficulties to the control algorithm that must include the pre-collision detection and convey this 
information to the operator. To tackle these constrains, a steering attractive force is set up to manipulate a robot arm and the three-dimensional force field is used to sense the arm's surrounding environments.

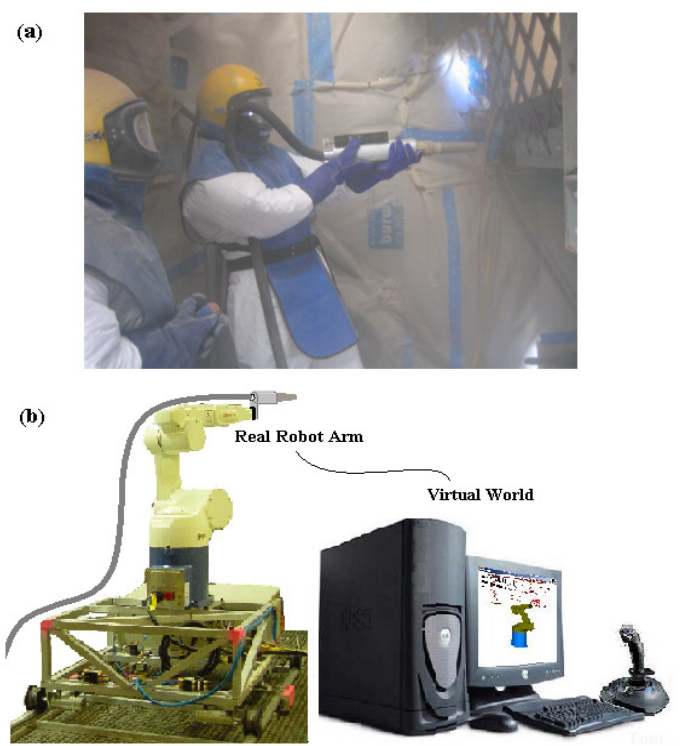

Figure 1. Grit blasting by manual operation (a), the proposed remote robotic system for grit blasting (b)

\section{VIRTUAL FORCE BASED ROBOT ARM MANIPULATION}

\subsection{Steering Attractive Force}

In grit blasting process, the operation is focused on the blasting stream and its blasting spot (target point). The nozzle movement has to be controlled on a configuration plane and the blasting stream will be considered as an orienting vector on the configuration plane. A force feedback joystick is incorporated to control the blasting spot and the joystick X-Y plane is matched to the blasting spot's configuration plane (see Fig. 2).

Given an instant target point of the blasting spot $\left(\mathbf{p}_{t}\right)$ and the position of the robot arm's end-effecter $\left(\mathbf{p}_{e}\right)$, a virtual attractive force that "pulls" the endeffecter to the target point will be generated. This attractive force can be defined by the distance $\left(S_{t}\right)$ between the current position of the manipulator's end-effecter and the target position.

$$
S_{t}=\left\|\mathbf{p}_{t}-\mathbf{p}_{e}\right\|
$$

The amplitude of the attractive force is limited by a force factor $\left(K_{a t t}\right)$ and given by a sigmoid function

$$
f_{\text {att }}=\frac{K_{a t t}}{1+K_{z}^{-1} e^{-K_{S} S_{t}}}
$$

where $K_{z}>0$ and $K_{s}$ are constants, which will determine how the attractive force varies with the distance between the end-effecter and the target point. The amplitude of the attractive force increases with the distance and its direction is from the current position of end-effecter to the target.

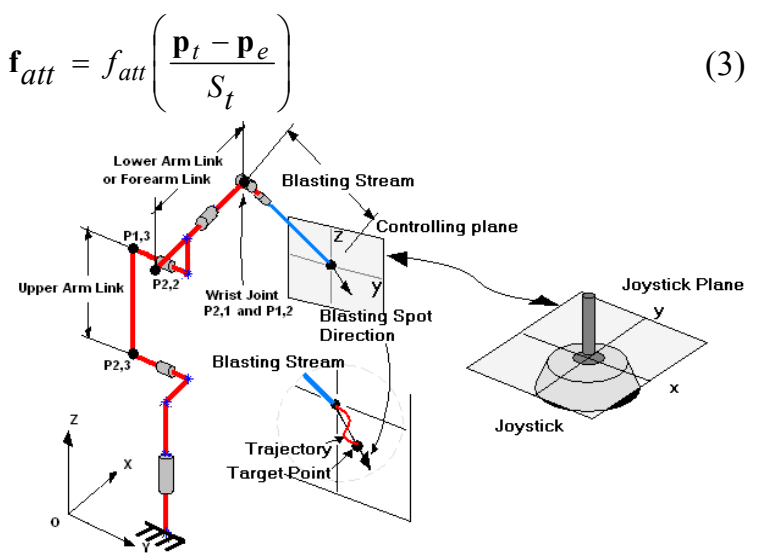

Figure 2. Definition of control and joystick coordinate plane parameters

\subsection{Definition of 3-dimensional Force Field}

This section introduces the $3 \mathrm{D}$ force field $\left(3 \mathrm{D}-\mathrm{F}^{2}\right)$ defined in[10][9]. The transformations from the robot's coordinate system to the global system are given by transfer function $\left({ }^{i} \mathbf{T}_{j}\right)$ and rotationtranslation metrics $\left({ }^{i} \mathbf{A}_{j}\right) . \mathbf{P}_{i(u, v, w)}$ and $\mathbf{P}_{i(x, y, z)}$ are points on the robot's coordinate system and the global system. Here

$$
\begin{aligned}
& { }^{0} \mathbf{T}_{n}=\prod_{m=0}^{n-1}{ }^{m} \mathbf{A}_{m+1} \\
& \mathbf{p}_{i(x, y, z)}={ }^{0} \mathbf{T}_{n} \mathbf{p}_{i(u, v, w)}, \text { where } \mathrm{i}=1,2
\end{aligned}
$$

To design the ellipsoid, $\mathrm{D}_{\min }$ and $\mathrm{D}_{\max }$ to cover a manipulator link, two points on a link are selected as the foci ( $\mathbf{p}_{1}$ and $\mathbf{p}_{2}$ in Fig. 3). To ensure that this 
ellipsoid will cover the whole body of the link, the length of major axis is set to $L K_{p}$, where $L$ is the distance between foci and $K_{p}>1$ is a constant. We define:

$$
C x=\frac{\left\|\mathbf{R}_{1}\right\|+\left\|\mathbf{R}_{2}\right\|}{L}
$$

where $\mathbf{R}_{1}, \mathbf{R}_{2}$ are the circles extended from the ellipsoid from the foci. To enlarge the ellipsoid, $K_{p}+E r$ is defined for $\mathrm{D}_{\max }$, where $E r>0$ is an allowance variable. Thus, the length of major axis of $\mathrm{D}_{\max }$ will be $\mathrm{L}\left(K_{p}+E r\right)$. For a point in $3 \mathrm{D}$ space ( $\mathbf{p}_{o b}$ in Fig. 3), if $\mathbf{p}_{o b}$ is on surface of $\mathrm{D}_{\min }, C x$ will equal to $K_{p}$. Moreover, if $\mathbf{p}_{o b}$ is on surface of $\mathrm{D}_{\max }$, $C x$ will equal to $K_{p}+E r$ as well. This can be defined regions on domain of $C x$ such that

$K p<C x<K p+E r$

By these regions, the sigmoid function is used to define the amplitude of repulsive force as

$$
f_{\text {rep }}=K f-\frac{K f}{1+e^{\left(-\frac{10(C x-K p-0.5 E r)}{E r}\right)}}
$$

where $K f$ is the maximum repulsive force. The repulsive force direction is defined as the unit vector that points from $\mathbf{p}_{o b}$ to $\mathbf{p}_{1}$.

$$
\begin{aligned}
& \mathbf{f}_{\text {rep }} \text { unit } \\
& \mathbf{f}_{\text {rep }}=f_{\text {rep }} \mathbf{f}_{\text {rep }}-\mathbf{p}_{1}-\mathbf{p}_{o b} \\
& \left\|\mathbf{p}_{1}-\mathbf{p}_{o b}\right\|
\end{aligned}
$$
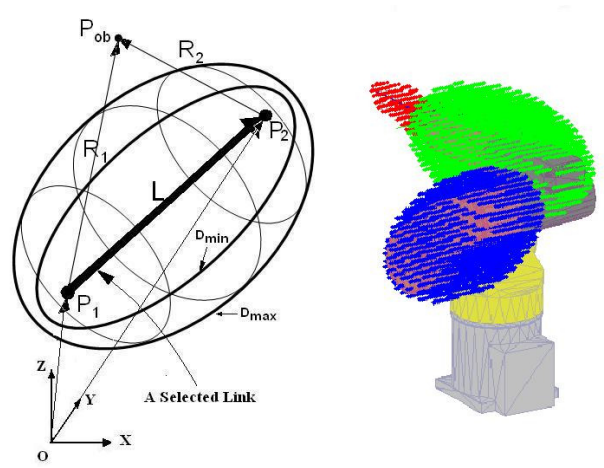

Figure 3. Parameters of $\mathrm{D}_{\min }$ and $\mathrm{D}_{\max }$ ellipsoid (left) and a robot arm covered by $\mathrm{D}_{\min }$ (right)

\section{DYNAMIC MODEL}

This section introduces how the attractive force and the $3 \mathrm{D}-\mathrm{F}^{2}$ method generate the robot arm's trajectory with real time collision avoidance. Since, the manipulator is driven by the torque at the joints, all forces will be converted to the torques by Jacobian matrices. In this paper, the virtual drive forces are calculated in two stages; the first to third joint $\mathbf{J}_{3}$ (arm motion) and the fourth to sixth joint rotation matrix (wrist motion).

For arm motion:

$\boldsymbol{\tau}_{\left(\theta_{1}, \theta_{2}, \theta_{3}\right)}=\mathbf{J}_{3}^{-1} \mathbf{f}_{\text {att }}+\sum_{i=1}^{3} \mathbf{J}_{i}^{-1} \mathbf{f}_{r e p_{i}}$

For wrist motion:

$\boldsymbol{\tau}_{\left(\theta_{4}, \theta_{5}\right)}=\left[{ }^{0} \mathbf{T}_{5}\right]^{-1} \mathbf{m}_{(\text {wrist })}$

where

$$
\begin{gathered}
\mathbf{m}_{(\text {wrist })}=\mathbf{l}_{(\text {stream })} \times \mathbf{f}_{\text {end }} \\
\mathbf{f}_{\text {end }}=\mathbf{f}_{\text {att }}+\mathbf{f}_{\text {rep3 } 3} \\
\mathbf{l}_{(\text {stream })}=\mathbf{p}_{\text {(blastingsopt })}-\mathbf{p}_{(\text {wrist })}
\end{gathered}
$$

The dynamics are

$$
\ddot{\mathbf{I}}=-\boldsymbol{\beta} \dot{\boldsymbol{\theta}}+\mathbf{K}_{\mathbf{m}} \boldsymbol{\tau}_{\left(\theta_{1}, \theta_{2}, \theta_{3}, \theta_{4}, \theta_{5}\right)}
$$

where, $\mathbf{K}_{m}$ is a motion-selecting matrix, which has three motion types; 1) arm motion, 2) wrist motion and 3) wrist-arm motion. $\boldsymbol{\beta}$ and $\mathbf{I}$ are a damping factor metric and an inertia metric of the robot arm, respectively.

Furthermore, the blasting tools; a hose and a nozzle are installed on the robot arm. The pressurized hose is rigid and difficult to be twisted. Thus, the sixth joint will stabilize the hose position, where it is always on the top position of robot arm, by rotating itself against the fourth joint. In order to hold the nozzle to a setting pose, the rotating speed of the sixth joint is set to opposite the rotating speed of the fourth joint.

$\dot{\theta}_{6}=-\dot{\theta}_{4}$

In every control cycle, the dynamic equations (1617) will give a set of joint's angles $(\boldsymbol{\theta})$ and send them to the robot controller.

In Fig 4, the joystick gives displacement outputs. $\mathbf{M}_{\mathrm{cp}}$ is a controlling plan's orientation matrix that will transfer the displacement signals from the 
joystick coordinate system to the desired controlling plan at the end-effecter of the robot arm. The transformed displacements are defined as the steering force obtained from the attractioon point, see Section 3.1. This force is applied into the robot arm dynamic to work out a instant pose of the robot arm. The pose is then fed back to determine the repulsive forces in $3 \mathrm{D}-\mathrm{F}^{2}$. The repulsive forces will push agaist the steering force and brake the robot arm before colliding onto an obstacle. After that, the collision-free pose will be transmitted to the robot arm's position controller. Besides, the repulsive forces are also sent back to the force feedback joystick to render the potential field arroud obstacles.
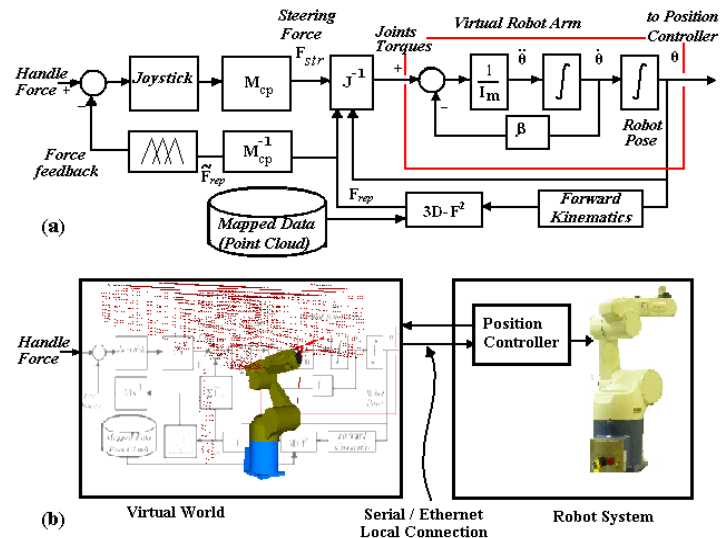

Figure 4. The system diagram and the robot arm system configuration

\section{SENSE OF TOUCH}

For the force feedback joystick, each axis of force input node needs two parameters: impulse force amplitude $\left(f_{\text {imp }}\right)$ and duration of zero order hold (zoh; $T_{d}$ ). Functionalizing these parameters can contribute to the sense of touch. Product of the force amplitude and $T_{d}$ is assumed as an impulse. The impulses will convey to operator's hand that the robot arm is approaching to an obstacle. Otherwise, the effect will decline when the robot arm has detracted from the obstacle. Thus, the impulse will be effective only when the direction of the force-field shielded link and its repulsive force direction are opposite. There are three repulsive forces (impulses), which could arise from the selected links, are generated separately. For the reason that the robot arm is manipulated on a controlling plane, all impulses should be transferred its coordinate system to the controlling plane coordinate. However, the joystick has only two axes of force feedback. Simple rules will be used to manage these force signals.

$\mathbf{f}_{i m p_{i}}=\left\{\begin{array}{cc}0, & \mathbf{f}_{a t t} \cdot \mathbf{f}_{r e p_{i}} \geq 0 \\ -K_{c} \mathbf{f}_{a t t}, & \mathbf{f}_{a t t} \cdot \mathbf{f}_{r e p_{i}}<0\end{array}\right.$

where $i=1,2,3$ ( 3 for the stream, 2 for the lowerarm and 1 for the upper-arm ellipsoid force field) and $K_{c}$ is a constant for scale the $\mathbf{f}_{\text {att }}$ to be a joystick force variable.

$$
\mathbf{f}_{i m p}=\left\{\begin{array}{lll}
\mathbf{f}_{i m p_{1}}, & T_{d}=0.01 s, & \mathbf{f}_{i m p_{1}}=\max \left(\mathbf{f}_{i m p_{i}}\right) \\
\mathbf{f}_{i m p_{2}}, & T_{d}=0.05 s, & \mathbf{f}_{i m p_{2}}=\max \left(\mathbf{f}_{i m p_{i}}\right) \\
\mathbf{f}_{i m p_{3}}, & T_{d}=0.10 s, & \mathbf{f}_{i m p_{3}}=\max \left(\mathbf{f}_{i m p_{i}}\right)
\end{array}\right.
$$

The $T_{d}$ will create the different feelings to operator's hand; short period of $T_{d}$ makes the operator feel a vibration. Otherwise, for longer $T_{d}$, the operator will feel a low magnitude ripple. The stiffness of joystick's handling depends on the amplitude of $\mathbf{f}_{\text {imp. }}$.

\section{EXPERIMENTS}

\subsection{System Implementation}

To prototype the remote operated robot arm system, a Virtual Robot for Grit Blasting Program has been written with algorithms of virtual force based approach. The program contains force feedback joystick interface, virtual reality (VR), a simulated robot arm, and a robot arm's position controller interface. The DirectX9 is used to interface the force feedback joystick. VR is programmed by using the OpenGL library.

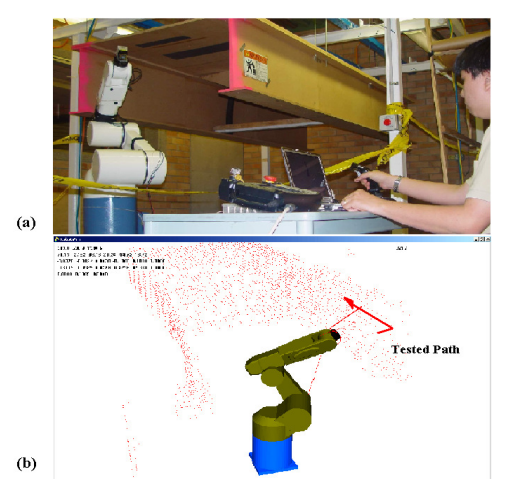

Figure 5. The prototype grit blasting robot (a), screenshot of the program under test (b) 

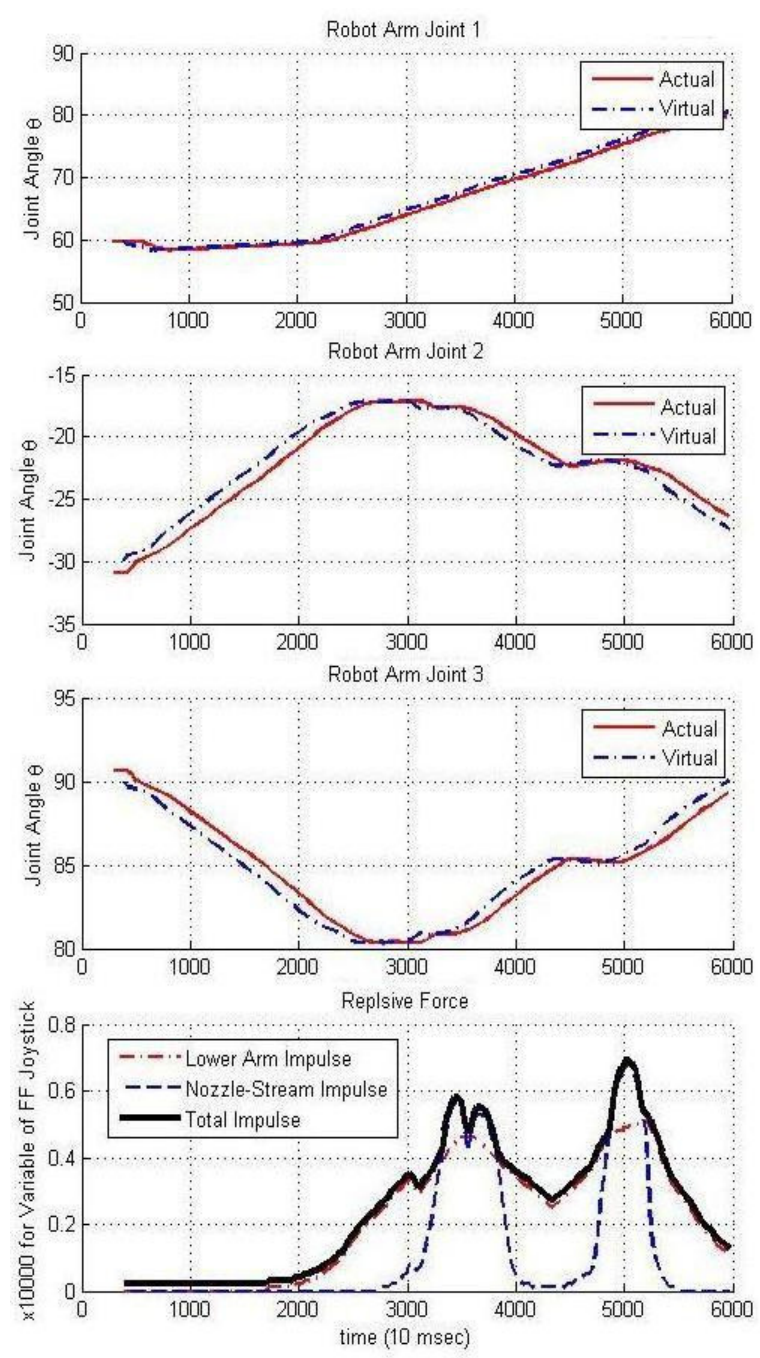

Figure 6. Test result of the first trial

The real-time thread cycle time is $10 \mathrm{~ms}$. The program runs on a $1.5 \mathrm{GHz}$ Pentium $\mathrm{M} \mathrm{PC}$ and is connected to the robot arm's controller through the serial port at 115,200 bps baud rate.

\subsection{Result}

The experiment is conducted to perform manual remote operations. An operator controls the robot arm approach to the I-beam of a bridge and directs the blasting stream on a surface of the I-beam (see path in Fig. 5). In the first trial, the speed of the robot
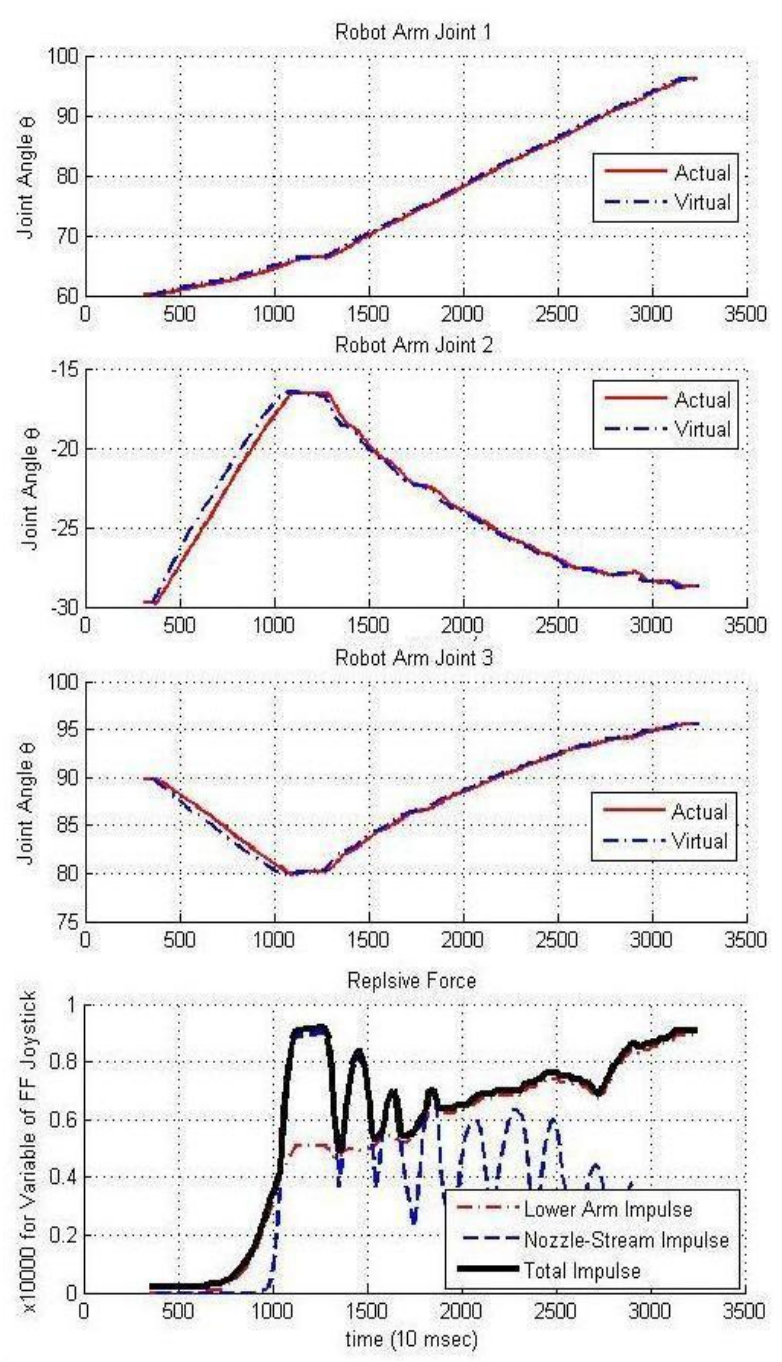

Figure 7. Test result of the second trial

arm (Denso VM6083) is set at $10 \%$ of its maximum speed and the second trial sets the speed at $30 \%$. The results in Fig. 6 and Fig. 7 compare the joint angles of robot arm for the virtual robot and the actual robot and the impulsive forces used for the operator's perception. The joint angle commands (dotted lines) send to the arm followed closely the derived angles (solid lines) from the actual arm. Force feedbacks reasonably represent the sense of touch conveyed to the operator. 


\subsection{Discussion}

In both tests, the arm follows almost the same path. Their start points are equal but the end of paths are just about the same because of manual control. Time delay increased when robot arm speed is set to the low speed. The fast motion caused the joystick to oscillate around the set point because the nearest obstacle is sorted from the group of closed points around the arm. Finally, the experiments showed that the collision avoidance are performed effectively.

\section{CONCLUSION}

This paper has presented a virtual force based approach for remote robot arm manipulation in the example grit blasting process. A force feedback joystick is used to issue controls to the arm and provides the sense of touch, on the obstacles, to the operator. This is achieved by using a virtual steering force to drive a virtual robot arm and reflection forces from obstacles generated from a 3-dimensional force field algorithm. Experimental results have indicated that the proposed technique is effective in performing remote robotic manipulations.

\section{ACKNOWLEDGEMENT}

This work is supported by the Australian Research Council (ARC) Linkage Grant (ARC-LP0776312), the ARC Centre of Excellence for Autonomous Systems (CAS) (funded by funded by the ARC and the New South Wales State Government), the Roads and Traffic Authority (RTA) and the University of Technology, Sydney. Mr. Pholchai Chotiprayanakul is supported by Thai Government Scholarship.

\section{REFERENCES}

[1] Y. Liao, et al. (2000), "Force Reflection and Manipulation for a VR-based Telerobotic System", Proceeding in National Science Council of ROC, Vol. 24, No. 5, 382-389.

[2] D. Constantinescu, et al. (2003), "Haptic Feedback using Local Models of Interaction", Proceedings of the 11th Symposium on Haptic Interfaces for Virtual Environment and Teleoperator Systems (HAPTICS'03), March 22-23,LosAngeles, USA, 416- 421
[3] D. Constantinescu, et al. (2004), "Haptic rendering of rigid body collisions", Proceedings of 12th International Symposium on Haptic Interfaces for Virtual Environment and Teleoperator Systems (HAPTICS'04), March 27-28, Chicago, Illinois, USA, 2-8.

[4] D. Constantinescu, et al. (2005), "Haptic rendering of rigid contacts using impulsive and penalty forces", IEEE Transactions on Robotics, Vol. 21, No. 3, June, 309-323.

[5] F. Nagata, et al. (2006), "Joystick Teaching System for Industrial Robots Using Fuzzy Compliance Control", Industrial Robotic Theory Modelling Control, Sam Cubero (Ed.) ISBN 3-86611-285-8, ARS/plV, Germany, December, 799-812.

[6] S. Kimand and M. Sitti (2006), "Task-Based and Stable Telenanomanipulation in a Nanoscale Virtual Environment", IEEE Transactions on Automation Science And Engineering, Vol. 3, No. 3, July, 240248.

[7] A. P. Shacklock, et al. (2007), "Intuitive Command of Manipulators in Microscale", Proceedings of. IEEE International Conference on Robotics and Automation, Roma, Italy, April 10-14, 846-851.

[8] J. R. Mckinsey and G. Chiu (2007), "Interfacing A Force-Feedback Joystick With A Hydraulic Robot Arm", Proceedings of the 2007 IEEE International Symposium on Computational Intelligence in Robotics and Automation, Jacksonville, FL, USA, June 20-23, 497-504.

[9] P. Chotiprayanakul, et al. (2007), "Collision-free trajectory planning for manipulators using virtual force based approach", Proceedings of the International Conference on Engineering, Applied Sciences, and Technology, Bangkok, Thailand, November 21-23, 6pp (CD-ROM).

[10] P. Chotiprayanakul, et al. (2007), “A 3-dimensional force field method for robot collision avoidance in complex environments", Proceedings of the 24th International Symposium on Automation and Robotics in Construction (ISARC 2007), Kochi, Kerala, India, September 19-21, 139-145. 